\title{
Lepton-Antineutrino Entanglement and Chiral Oscillations
}

\author{
Victor A. S. V. Bittencourt ${ }^{1, * \mathbb{D}}$, Alex E. Bernardini ${ }^{2}$ and Massimo Blasone ${ }^{3,4} \mathbb{D}$ \\ 1 Max Planck Institute for the Science of Light, Staudtstr. 2, PLZ 91058 Erlangen, Germany \\ 2 Departamento de Física, Universidade Federal de São Carlos, P.O. Box 676, \\ São Carlos 13565-905, São Paulo, Brazil; alexeb@ufscar.br \\ 3 Dipartimento di Fisica, Università degli Studi di Salerno, Via Giovanni Paolo II, 132, 84084 Fisciano, Italy; \\ mblasone@unisa.it \\ 4 INFN, Sezioni di Napoli, Gruppo Collegato di Salerno, 80126 Naples, Italy \\ * Correspondence: victor.bittencourt@mpl.mpg.de
}

Citation: Bittencourt, V.A.S.V.;

Bernardini, A.E.; Blasone, M.

Lepton-Antineutrino Entanglement and Chiral Oscillations. Universe 2021, 7, 293. https://doi.org/10.3390/ universe7080293

Academic Editor: Julio Marny Hoff da Silva

Received: 13 July 2021

Accepted: 5 August 2021

Published: 9 August 2021

Publisher's Note: MDPI stays neutral with regard to jurisdictional claims in published maps and institutional affiliations.

Copyright: (C) 2021 by the authors. Licensee MDPI, Basel, Switzerland. This article is an open access article distributed under the terms and conditions of the Creative Commons Attribution (CC BY) license (https:// creativecommons.org/licenses/by/ $4.0 /)$.

\begin{abstract}
Dirac bispinors belong to an irreducible representation of the complete Lorentz group, which includes parity as a symmetry yielding two intrinsic discrete degrees of freedom: chirality and spin. For massive particles, chirality is not dynamically conserved, which leads to chiral oscillations. In this contribution, we describe the effects of this intrinsic structure of Dirac bispinors on the quantum entanglement encoded in a lepton-antineutrino pair. We consider that the pair is generated through weak interactions, which are intrinsically chiral, such that in the initial state the lepton and the antineutrino have definite chirality but their spins are entangled. We show that chiral oscillations induce spin entanglement oscillations and redistribute the spin entanglement to chiralityspin correlations. Such a phenomenon is prominent if the momentum of the lepton is comparable with or smaller than its mass. We further show that a Bell-like spin observable exhibits the same behavior of the spin entanglement. Such correlations do not require the knowledge of the full density matrix. Our results show novel effects of the intrinsic bispinor structure and can be used as a basis for designing experiments to probe chiral oscillations via spin correlation measurements.
\end{abstract}

Keywords: entanglement; bispinors; chirality

\section{Introduction}

To ensure that transformations between inertial frames preserve probabilities, Wigner proposed in his seminal work [1] that states describing particles should belong to unitary irreducible representations (irreps) of the Poincaré group. In such a framework, the degrees of freedom that a particle carries are defined by the particular irrep in question. Specifically, the irreps of the Poincaré group are classified by its Casimir invariants: momentum (mass) and the eigenvalues of the Pauli-Lubanski vector, related to the spin [2]. This description of particle states in terms of irreps of the Poincaré group has been used not only in particle and high energy physics [3] but also in connection with information theory for studying transformation properties of quantum correlations [4-7] and for quantum protocols in relativistic setups, such as clock synchronization [8,9] and teleportation [10].

Another prominent example in the context of relativistic quantum mechanics is the Dirac bispinor, which belongs to an irrep of the complete Lorentz group. This group includes parity as a symmetry [2], which connects the two irreps of the (proper) Lorentz group, given in terms of the two-component Weyl spinors. The irreps of the complete Lorentz group are given in terms of a $S U(2) \otimes S U(2)$ group structure, associated with two discrete degrees of freedom [2,11-13]: spin and chirality (or intrinsic parity). Since a state described by a Dirac bispinor has two dichotomic degrees of freedom, it can be understood as a two-qubit state $[11,12]$. In general, chirality and spin of a single particle can become entangled under external potentials [14], as noticed for Dirac-like systems, such as bilayer graphene [15].

The intrinsic structure of the Dirac bispinor has also implications for the dynamics of free particles. Helicity (the projection of spin in the direction of the momentum) is a 
conserved quantity, but chirality is not [16]. In fact, the different chiral components of a bispinor are coupled via the mass term of the Dirac Hamiltonian. This generates chiral oscillations [17], which can be related to the Zitterbewegung effect [18]. The dynamical features of chiral oscillations are particularly relevant for dynamics of neutrinos [19-21]. Neutrinos and antineutrinos only interact via weak processes, which are inherently chiral [22] and create such particles in states with definite chirality. Under free dynamics, such massive (anti)neutrino states exhibit a finite probability of being in the opposite chirality. The amplitude of these oscillations depends on the ratio between mass and energy and is prominent in the non-relativistic dynamical regime [23]. Since only one chiral component is measurable through weak processes, chiral oscillations can yield a depletion of the measured flux of the cosmic neutrino background [24-26].

In this contribution, we show that chiral oscillations affect quantum correlations shared between spins of a lepton-antineutrino pair. We consider a singlet state of a lepton and a massive antineutrino propagating along opposite directions. Assuming that such state is created by weak interactions (e.g., by the decay of a pion, as seen in the pion's rest frame), the massive antineutrino and the lepton have, at $t=0$, definite chiralities, modeled here by a chiral projection of the Dirac bispinors associated with the antineutrino and with the lepton. The spins are in a perfect anticorrelated spin state, as depicted in Figure 1. Under free evolution, chiral oscillations change the superposition form of the initial state, inducing oscillations on the correlations shared by the spins. We compute such oscillations both for the quantum entanglement encoded in the spins [27] and for Bell-type spin correlations [28].

(a)

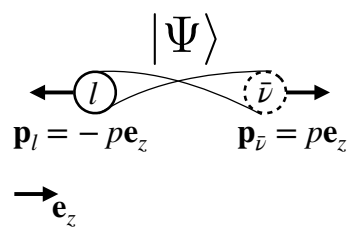

(b)

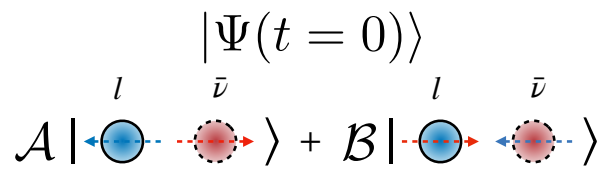

(c)

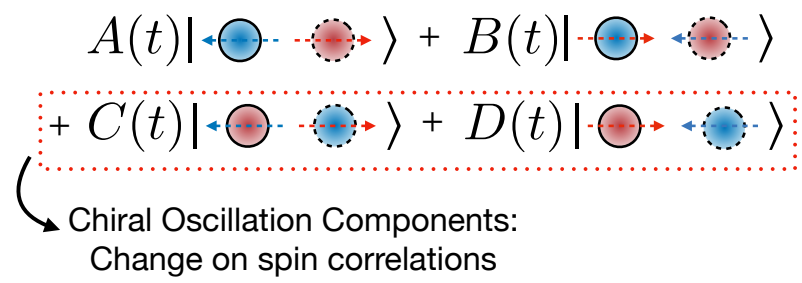

Figure 1. Framework: (a) a lepton and an antineutrino propagate in opposite directions with same momentum. Each is described by a Dirac bispinor, carrying spin and chirality degrees of freedom. (b) At $t=0$, the state is a superposition in which the lepton and the antineutrino have definite chiralities. The coefficients $\mathcal{A}$ and $\mathcal{B}$ depend on the masses of the particles and on the momentum. (c) Under free evolution, the chiralities oscillate, changing the initial superposition and the correlations shared between the spins.

We show that chiral oscillations induce a redistribution of entanglement from spinspin to spin-chirality. As a consequence, the amount of entanglement encoded only on the spins of the pair oscillates in time with a frequency that depends on the dynamical regime of propagation of both particles. In fact, even in the configuration where the lepton mass is much larger than the antineutrino mass, oscillations of the spin-spin entanglement are still relevant provided that the lepton is in the non-relativistic dynamical regime. Such entanglement oscillations exhibit a maximum amplitude for momenta of the order of the antineutrino mass, a resonance-like behavior. The spin-spin Bell-type correlation exhibits the same behavior of the spin-spin entanglement, but since its measurement requires only specific spin-spin correlations (and not a full reconstruction of the density matrix), it is more convenient for experiments. 


\section{Chiral Oscillations and Spin-Spin Entanglement}

We consider the dynamics of a state describing a lepton-antineutrino pair propagating in opposite directions with the same momentum. We assume that the lepton (hereafter indicated by the subscript $l$ ) with mass $m_{l}$ has momentum $-p \mathbf{e}_{z}$, while the antineutrino (indicated by the subscript $\bar{v}$ ) with mass $m_{\bar{v}}$ has momentum $p \mathbf{e}_{z}$, as depicted in Figure 1. As a toy model for the creation process, we assume the following superposition

$$
|\Phi\rangle=\frac{\left|v_{\uparrow}\left(p, m_{\bar{v}}\right)\right\rangle \otimes\left|u_{\downarrow}\left(-p, m_{l}\right)\right\rangle-\left|v_{\downarrow}\left(p, m_{\bar{v}}\right)\right\rangle \otimes\left|u_{\uparrow}\left(-p, m_{l}\right)\right\rangle}{\sqrt{2}}
$$

where $\left|u_{\uparrow(\downarrow)}(p, m)\right\rangle$ and $\left|v_{\uparrow(\downarrow)}(p, m)\right\rangle$ denote the Dirac bispinors with parallel $(\uparrow)$ and antiparallel $(\downarrow)$ spins polarized along $\mathbf{e}_{z}$. Those bispinors are the eigenstates of the free Dirac Hamiltonian

$$
\hat{H}=\hat{\mathbf{p}} \cdot \hat{\boldsymbol{\alpha}}+m \hat{\beta},
$$

with energies $\pm E_{p, m}= \pm \sqrt{p^{2}+m^{2}}$, where neutral units $c=\hbar=1$ have been considered, boldface letters indicate vectors, and hats "^" denote operators. The $4 \times 4$ Dirac matrices $\hat{\alpha}$ and $\hat{\beta}$ satisfy the anti-commutation relations $\hat{\alpha}_{i} \hat{\alpha}_{j}+\hat{\alpha}_{j} \hat{\alpha}_{i}=2 \delta_{i j} \hat{I}_{4}, \hat{\alpha}_{i} \hat{\beta}+\hat{\beta} \hat{\alpha}_{i}=0$, and $\hat{\beta}^{2}=\hat{I}_{4}$, where $\hat{I}_{N}$ denotes the $N \times N$ identity matrix. We adopt the chiral representation of the Dirac matrices [29]

$$
\hat{\alpha}_{i}=\left[\begin{array}{cc}
\hat{\sigma}_{i} & 0 \\
0 & -\hat{\sigma}_{i}
\end{array}\right], \quad \hat{\beta}=\left[\begin{array}{cc}
0 & \hat{I}_{2} \\
\hat{I}_{2} & 0
\end{array}\right],
$$

with $\hat{\sigma}_{i}$ denoting the Pauli matrices, which returns for the bispinors

$$
\begin{array}{rlrl}
\left|u_{\uparrow}(p, m)\right\rangle & =N_{p, m}\left[\begin{array}{cl}
f_{+}(p, m)|\uparrow\rangle \\
f_{-}(p, m)|\uparrow\rangle
\end{array}\right], & \left|u_{\downarrow}(p, m)\right\rangle=N_{p, m}\left[\begin{array}{l}
f_{-}(p, m)|\downarrow\rangle \\
f_{+}(p, m)|\downarrow\rangle
\end{array}\right], \\
\left|v_{\uparrow}(p, m)\right\rangle=N_{p, m}\left[\begin{array}{c}
f_{+}(p, m)|\uparrow\rangle \\
-f_{-}(p, m)|\uparrow\rangle
\end{array}\right], & \left|v_{\downarrow}(p, m)\right\rangle=N_{p, m}\left[\begin{array}{c}
f_{-}(p, m)|\downarrow\rangle \\
-f_{+}(p, m)|\downarrow\rangle
\end{array}\right],
\end{array}
$$

where $|\uparrow\rangle$ and $|\downarrow\rangle$ are the eigenstates of $\hat{\sigma}_{z}$, and the short hand notation sets

$$
\begin{array}{r}
N_{p, m}=\sqrt{\frac{E_{p, m}+m}{4 E_{p, m}}} \\
f_{ \pm}(p, m)=1 \pm \frac{p}{E_{p, m}+m} .
\end{array}
$$

Finally, the normalization of the bispinors is $\left\langle u_{s}(p, m) \mid u_{l}(p, m)\right\rangle=\left\langle v_{s}(p, m) \mid v_{l}(p, m)\right\rangle=\delta_{s l}$, and the orthogonality relations are $\left\langle u_{s}(p, m) \mid v_{l}(-p, m)\right\rangle=0$.

The choice of the singlet state (1) is motivated by the decay of a pion into a leptonantineutrino pair in the center of mass of the pion [30-32], a process generated by weak interactions. Since weak interactions are inherently chiral and select definite chiral components of the bispinors, we model such an effect by projecting the state (1) into definite chiralities: the antineutrino into right (positive) chirality and the lepton into left (negative) chirality, such that, at $t=0$, the state is given by

$$
|\Psi(0)\rangle=\frac{\hat{\Pi}_{R}^{(\bar{v})} \otimes \hat{\Pi}_{L}^{(l)}|\Phi\rangle}{\left\langle\Phi\left|\hat{\Pi}_{R}^{(\bar{v})} \otimes \hat{\Pi}_{L}^{(l)}\right| \Phi\right\rangle} .
$$

The chirality projectors are given in terms of the chiral matrix $\hat{\gamma}_{5}=\operatorname{diag}\left[\hat{I}_{2},-\hat{I}_{2}\right]$ by

$$
\hat{\Pi}_{R(L)}^{(A)}=\frac{\hat{I}^{(A)}+(-) \hat{\gamma}_{5}^{(A)}}{2}, \quad(A=\bar{v}, l)
$$


such that

$$
|\Psi(0)\rangle=\mathcal{A}\left(p, m_{l}, m_{\bar{v}}\right)\left|\bar{v}_{\uparrow}(0)\right\rangle \otimes\left|l_{\downarrow}(0)\right\rangle-\mathcal{B}\left(p, m_{l}, m_{\bar{v}}\right)\left|\bar{v}_{\downarrow}(0)\right\rangle \otimes\left|l_{\uparrow}(0)\right\rangle .
$$

The chirality projected states at $t=0$ are

$$
\left|\bar{v}_{\uparrow(\downarrow)}(0)\right\rangle=\left[\begin{array}{c}
|\uparrow(\downarrow)\rangle \\
0
\end{array}\right], \quad\left|l_{\uparrow(\downarrow)}(0)\right\rangle=\left[\begin{array}{c}
0 \\
|\uparrow(\downarrow)\rangle
\end{array}\right],
$$

and the coefficients of the superposition are given by

$$
\begin{aligned}
& \mathcal{A}\left(p, m_{l}, m_{\bar{v}}\right)=N_{p, m_{l}} N_{p, m_{\bar{v}}} f_{+}\left(p, m_{\bar{v}}\right) f_{-}\left(p, m_{l}\right)\left[\frac{1}{2}-\frac{p^{2}}{2 E_{p, m_{l}} E_{p, m_{\bar{v}}}}\right]^{-\frac{1}{2}}, \\
& \mathcal{B}\left(p, m_{l}, m_{\bar{v}}\right)=N_{p, m_{l}} N_{p, m_{\bar{v}}} f_{-}\left(p, m_{\bar{v}}\right) f_{+}\left(p, m_{l}\right)\left[\frac{1}{2}-\frac{p^{2}}{2 E_{p, m_{l}} E_{p, m_{\bar{v}}}}\right]^{-\frac{1}{2}} .
\end{aligned}
$$

If $p \gg m_{\bar{v}}$, then $\mathcal{A} \gg \mathcal{B}$, and the largest contribution to the superposition (8) is the first term.

Dirac bispinors belong to an irreducible representation of the complete Lorentz group, and as such they carry two intrinsic dichotomic degrees of freedom [2]: chirality (or intrinsic parity) and spin. The former degree of freedom is related to the inclusion of spatial parity as a symmetry. In this framework, we can understand each single particle state as a two qubit state [11], and a bispinor state belongs to a composite Hilbert space $\mathcal{H}_{S} \otimes \mathcal{H}_{C}$ with $\operatorname{dim}\left[\mathcal{H}_{C}\right]=\operatorname{dim}\left[\mathcal{H}_{S}\right]=2$. Here, $\mathcal{H}_{S(C)}$ is associated with the spin (chirality) degree of freedom. Therefore, a two particle state, such as (1), can be interpreted as a four qubit state [13]. The quantum bits are chirality and spin of the antineutrino $\left(C_{\bar{v}}\right.$ and $S_{\bar{v}}$, respectively) and chirality and spin of the lepton $\left(C_{l}\right.$ and $S_{l}$, respectively). The state of the lepton-antineutrino pair is then described in the composite Hilbert space $\mathcal{H}_{C_{\bar{v}}} \otimes \mathcal{H}_{S_{\bar{v}}} \otimes \mathcal{H}_{C_{l}} \otimes \mathcal{H}_{S_{l}}$.

The superposition (1) is an entangled state: it can not be written as the tensor product of pure states $\left|\psi_{C_{\bar{v}}}\right\rangle \otimes\left|\psi_{S_{\bar{v}}}\right\rangle \otimes\left|\psi_{C_{l}}\right\rangle \otimes\left|\psi_{S_{l}}\right\rangle$, where $\left|\psi_{A}\right\rangle \in \mathcal{H}_{A}$ with $A$ denoting the degrees of freedom. The entanglement encoded in (1) involves all four degrees of freedom of the state. On the other hand, the chiral projected state (8), describing the intrinsic chiral character of weak interactions, encodes entanglement only between the spins. In fact, we can readily write $|\Psi(0)\rangle=\left|+_{C_{\bar{\nu}}}\right\rangle \otimes\left|-C_{l}\right\rangle \otimes\left|\Psi_{S_{\bar{v}}, S_{l}}\right\rangle$, with $\left| \pm_{A}\right\rangle$ denoting the positive (negative) chirality of $A=C_{\bar{v}, l}$, and

$$
\left|\Psi_{S_{\bar{v}}, S_{l}}\right\rangle=\mathcal{A}\left(p, m_{l}, m_{\bar{v}}\right)\left|\uparrow_{S_{\bar{v}}}\right\rangle \otimes\left|\downarrow_{S_{l}}\right\rangle-\mathcal{B}\left(p, m_{l}, m_{\bar{v}}\right)\left|\downarrow_{S_{\bar{v}}}\right\rangle \otimes\left|\uparrow_{S_{l}}\right\rangle
$$

is the joint spin state at $t=0$.

To compute the amount of entanglement shared between the spins, we adopt the entanglement quantifier called negativity [33,34]. The Peres separability criterion [33] states that the partial transposed density matrix of a separable state has only positive eigenvalues. For a two-qubit state, partial transposition corresponds to the transformation $|i j\rangle\langle k l| \rightarrow$ $|i l\rangle\langle k j|$, i.e., it is a transposition on the subspace of only one of the subsystems. The criterion allows the definition of the negativity as an entanglement quantifier for two-qubit states: given a density matrix $\varrho$ representing the state, it is defined as $\mathcal{N}_{S_{\bar{\nu}}, S_{l}}[\varrho]=\left\|\varrho^{T}\right\|-1$, where $\left\|\varrho^{T}\right\|$ is the trace norm of the partial transposed matrix $\varrho^{T}$, given in terms of its eigenvalues $\lambda_{i}$ by $\left\|\varrho^{T}\right\|=\sum_{i}\left|\lambda_{i}\right|$. This entanglement quantifier is valid for both pure and mixed states [27]. 
We can now calculate the entanglement shared between the spins by first evaluating the spin-reduced density matrix:

$$
\begin{aligned}
& \rho_{S_{\bar{v}}, S_{l}}(0)=\operatorname{Tr}_{C_{l}, C_{\bar{v}}}[|\Psi(0)\rangle\langle\Psi(0)|]=\left|\Psi_{S_{\bar{v}}, S_{l}}\right\rangle\left\langle\Psi_{S_{\bar{v}}, S_{l}}\right| \\
& =\mathcal{A}^{2}\left(p, m_{l}, m_{\bar{v}}\right)\left|\uparrow_{\bar{v} \downarrow_{l}}\right\rangle\left\langle\uparrow_{\bar{v} \downarrow_{l}}\left|+\mathcal{B}^{2}\left(p, m_{l}, m_{\bar{v}}\right)\right| \downarrow_{\bar{v}} \uparrow_{l}\right\rangle\left\langle\downarrow_{\bar{v}} \uparrow_{l}\right|
\end{aligned}
$$

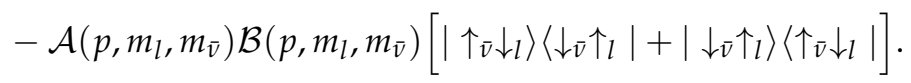

Partial transposition yields $\rho_{S_{\bar{v}}, S_{l}}^{T}$ from which we obtain the spin-spin negativity for the state at $t=0$

$$
\mathcal{N}_{S_{\bar{v}}, S_{l}}(0) \equiv \mathcal{N}\left[\rho_{S_{\bar{v}}, S_{l}}(0)\right]=2\left|\mathcal{A}\left(p, m_{l}, m_{\bar{v}}\right) \mathcal{B}\left(p, m_{l}, m_{\bar{v}}\right)\right|
$$

Figure 2 depicts $\mathcal{N}_{S_{l}, S_{\bar{v}}}(0)$ as a function of the momentum $p$ and the lepton mass $m_{l}$ in units of the antineutrino mass $m_{\bar{v}}$. The amount of entanglement depends both on the dynamical regime and on the lepton-antineutrino mass ratio. For $m_{l} \gg m_{\bar{v}}$ and $p \gg m_{l, \bar{v}}$, the spin-spin entanglement vanishes. In this limit, the antineutrino is ultrarelativistic, and thus its chirality equals its helicity. In terms of (8), $\mathcal{A} \gg \mathcal{B}$ in this limit, and the state becomes separable. In a pion decay process, this is expected due to the small mass of antineutrinos. One can also understand the separability of the state in terms of conservation of angular momentum: the initial pion has zero spin; therefore, since ultra-relativistic neutrinos have a definite helicity, the spin-polarization of the lepton is fixed [30]. Furthermore, the entanglement between spins is more prominent for $m_{v}>p$, even if $m_{\bar{v}} \ll m_{l}$.

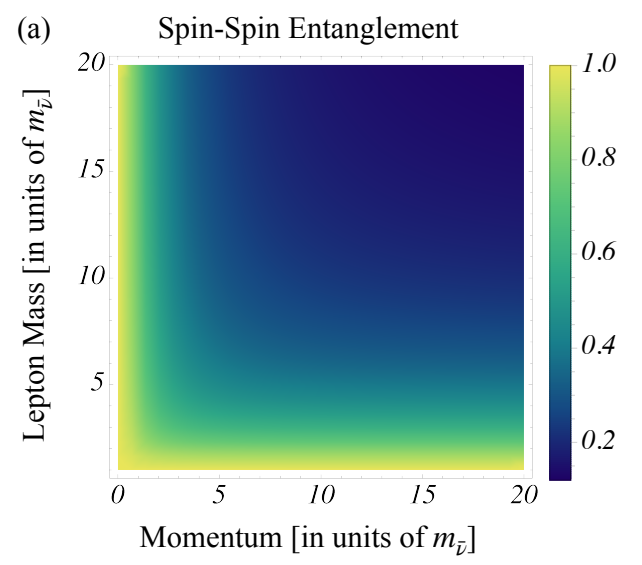

(b)

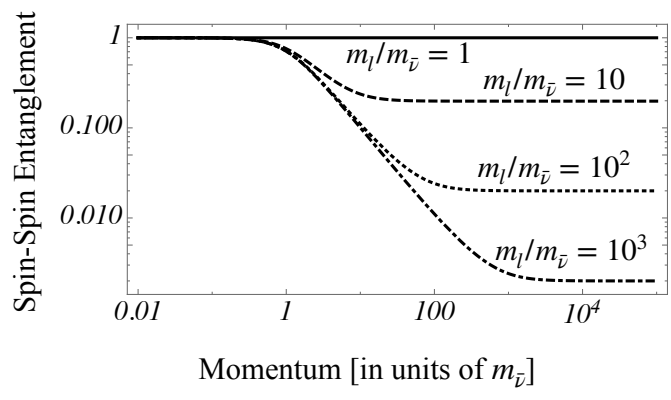

Figure 2. (a) Entanglement between the lepton and the antineutrino spins in the initial state (8) as a function of the momentum and of the lepton mass (in units of the antineutrino mass). (b) Same as (a) but for specific lepton-antineutrino mass ratios as a function of the momentum (in log-log scale).

Since chirality is not a conserved quantity under the free Dirac equation, temporal evolution can induce chiral oscillations $[17,18]$. For a two-particle state such as (8), this yields a change in the form of the superposition that dynamically redistributes the quantum correlations, encoded initially only between the spins, to other partitions of the system. Thus, because both particles are massive and described by Dirac bispinors, time evolution induces entanglement oscillations whose characteristics are intrinsically linked to those of the chiral oscillations.

For any given bispinor $|w(0)\rangle$, its temporal evolution can be obtained by a decomposition into the Dirac bispinors as $[29,35]$

$$
|w(t)\rangle=\sum_{s=\uparrow, \downarrow} U_{w, s} e^{-i E_{p, m} t}\left|u_{s}(p, m)\right\rangle+V_{w, s} e^{i E_{p, m} t}\left|v_{s}(-p, m)\right\rangle,
$$


where $U_{w, s}=\left\langle u_{s}(p, m) \mid w(0)\right\rangle$ and $V_{w, s}=\left\langle v_{s}(-p, m) \mid w(0)\right\rangle$. For the joint lepton-antineutrino state, we obtain

$$
|\Psi(t)\rangle=\mathcal{A}\left(p, m_{l}, m_{\bar{v}}\right)\left|\bar{v}_{\uparrow}(t)\right\rangle \otimes\left|l_{\downarrow}(t)\right\rangle-\mathcal{B}\left(p, m_{l}, m_{\bar{v}}\right)\left|\bar{v}_{\downarrow}(t)\right\rangle \otimes\left|l_{\uparrow}(t)\right\rangle,
$$

where the antineutrino components are given by

$$
\begin{aligned}
\left|\bar{v}_{\uparrow}(t)\right\rangle & =\mathcal{N}_{p, m_{\bar{v}}}\left[e^{-i E_{p, m_{\bar{v}}} t} f_{+}\left(p, m_{\bar{v}}\right)\left|u_{\uparrow}\left(p, m_{\bar{v}}\right)\right\rangle+e^{i E_{p, m_{\bar{v}}} t} f_{-}\left(p, m_{\bar{v}}\right)\left|v_{\uparrow}\left(-p, m_{\bar{v}}\right)\right\rangle\right], \\
\left|\bar{v}_{\downarrow}(t)\right\rangle & =\mathcal{N}_{p, m_{\bar{v}}}\left[e^{-i E_{p, m_{\bar{v}}} t} f_{-}\left(p, m_{\bar{v}}\right)\left|u_{\downarrow}\left(p, m_{\bar{v}}\right)\right\rangle+e^{i E_{p, m_{\bar{v}}} t} f_{+}\left(p, m_{\bar{v}}\right)\left|v_{\downarrow}\left(-p, m_{\bar{v}}\right)\right\rangle\right],
\end{aligned}
$$

and the lepton components are

$$
\begin{aligned}
& \left|l_{\uparrow}(t)\right\rangle=\mathcal{N}_{p, m_{l}}\left[e^{-i E_{p, m_{l}} t} f_{+}\left(p, m_{l}\right)\left|u_{\uparrow}\left(-p, m_{l}\right)\right\rangle-e^{i E_{p, m_{l}} t} f_{-}\left(p, m_{l}\right)\left|v_{\uparrow}\left(p, m_{l}\right)\right\rangle\right], \\
& \left|l_{\downarrow}(t)\right\rangle=\mathcal{N}_{p, m_{l}}\left[e^{-i E_{p, m_{l}} t} f_{-}\left(p, m_{l}\right)\left|u_{\downarrow}\left(-p, m_{l}\right)\right\rangle-e^{i E_{p, m_{l}} t} f_{+}\left(p, m_{l}\right)\left|v_{\downarrow}\left(p, m_{l}\right)\right\rangle\right] .
\end{aligned}
$$

With the time-evolved state (15), we can now compute the quantities of interest: the spin-spin entanglement and the average chiralities of the lepton and of the antineutrino. Since $\left\langle\bar{v}_{i}(t) \mid \bar{v}_{j}(t)\right\rangle=\left\langle l_{i}(t) \mid l_{j}(t)\right\rangle=\delta_{i j}(\{i, j\}=\uparrow, \downarrow)$, we obtain the reduced density matrices for the antineutrino and the lepton by

$$
\begin{aligned}
\rho_{\bar{v}}(t) & =\operatorname{Tr}_{l}[|\Psi(t)\rangle\langle\Psi(t)|] \\
& =\mathcal{A}^{2}\left(p, m_{l}, m_{\bar{v}}\right)\left|\bar{v}_{\uparrow}(t)\right\rangle\left\langle\bar{v}_{\uparrow}(t)\left|+\mathcal{B}^{2}\left(p, m_{l}, m_{\bar{v}}\right)\right| \bar{v}_{\downarrow}(t)\right\rangle\left\langle\bar{v}_{\downarrow}(t)\right|, \\
\rho_{l}(t) & =\operatorname{Tr}_{\bar{v}}[|\Psi(t)\rangle\langle\Psi(t)|] \\
& =\mathcal{A}^{2}\left(p, m_{l}, m_{\bar{v}}\right)\left|l_{\downarrow}(t)\right\rangle\left\langle l_{\downarrow}(t)\left|+\mathcal{B}^{2}\left(p, m_{l}, m_{\bar{v}}\right)\right| l_{\uparrow}(t)\right\rangle\left\langle l_{\uparrow}(t)\right| .
\end{aligned}
$$

We first notice that $\rho_{\bar{v}, l}(t)$ are mixed states:

$$
\begin{aligned}
\operatorname{Tr}\left[\rho_{\bar{v}}^{2}(t)\right]=\operatorname{Tr}\left[\rho_{l}^{2}(t)\right] & =\mathcal{A}^{4}\left(p, m_{l}, m_{\bar{v}}\right)+\mathcal{B}^{4}\left(p, m_{l}, m_{\bar{v}}\right) \\
& =1-\frac{\mathcal{N}_{S_{\bar{v}}, S_{l}}^{2}(0)}{2}<1 .
\end{aligned}
$$

Since the evolution is unitary, the degree of mixedness $\operatorname{Tr}\left[\rho_{\bar{v}, l}^{2}(t)\right]$ is time-independent. The joint state (15) is pure at all times; therefore, (19) computes the total amount of entanglement encoded in the bipartition $\left(C_{\bar{v}}, S_{\bar{v}}\right) ;\left(C_{l}, S_{l}\right)$ [36], that is, the entanglement between all the degrees of freedom of the antineutrino as a whole and all the degrees of freedom of the lepton as a whole. Such entanglement is constant in time and given in terms of the initial spin-spin entanglement via the term $\propto \mathcal{N}_{S_{\bar{v}}, S_{l}}(0)$ (see also Equation (13)). The average chiralities of the antineutrino and lepton are given by $\left\langle\hat{\gamma}_{5}\right\rangle_{A}(t)=\operatorname{Tr}_{A}\left[\hat{\gamma}_{5}^{(A)} \rho_{A}(t)\right]$ with $A=\bar{v}, l$ and read

$$
\begin{aligned}
& \left\langle\hat{\gamma}_{5}\right\rangle_{\bar{v}}(t)=1-\frac{m_{\bar{v}}^{2}}{E_{p, m_{\bar{v}}}^{2}}\left[1-\cos \left(2 E_{p, m_{\bar{v}}} t\right)\right], \\
& \left\langle\hat{\gamma}_{5}\right\rangle_{l}(t)=-1+\frac{m_{l}^{2}}{E_{p, m_{l}}^{2}}\left[1-\cos \left(2 E_{p, m_{l}} t\right)\right] .
\end{aligned}
$$

Different from (8), it is not possible to write $|\Psi(t)\rangle=\left|\psi_{C_{\bar{v}}}(t)\right\rangle \otimes\left|\psi_{C_{l}}(t)\right\rangle \otimes\left|\psi_{S_{\bar{v}}, S_{l}}(t)\right\rangle$, that is, the chiralities and the spins become entangled. The free evolution under the Dirac equation induces oscillations between left- and right-handed chiralities for both antineutrino and lepton, which changes the initial superposition and redistributes the correlation content carried by the state. The density matrix of the spins $\rho_{S_{\bar{v}}, S_{l}}(t)=\operatorname{Tr}_{\text {Chirality }}[|\Psi(t)\rangle\langle\Psi(t)|]$ 
is a mixed state with entanglement dynamics directly affected by chiral oscillations. The entanglement between the spins is again evaluated in terms of the negativity:

$$
\mathcal{N}_{S_{\bar{v}}, S_{l}}(t) \equiv \mathcal{N}\left[\rho_{S_{\bar{v}}, S_{l}}(t)\right]=\left\|\rho_{S_{\bar{v}}, S_{l}}^{T}(t)\right\|-1=\mathcal{N}_{S_{\bar{\nu}}, S_{l}}(0) \Gamma(t)
$$

with the time-dependent factor given in terms of the chiralities (20) as

$$
\Gamma(t)=\sum_{j=\bar{v}, l}\left[1-\frac{p^{2}}{m_{j}^{2}}\left(\left\langle\hat{\gamma}_{5}\right\rangle_{j}(t)-1\right)^{2}\right]^{\frac{1}{2}} .
$$

The degree of mixedness of the spin density matrix reads

$$
\operatorname{Tr}\left[\rho_{S_{\bar{\nu}}, S_{l}}^{2}(t)\right]=1-\frac{\mathcal{N}_{S_{\bar{\nu}}, S_{l}}^{2}(0)\left(1-|\Gamma(t)|^{2}\right)}{2},
$$

which quantifies the entanglement in the bipartition $\left(S_{\bar{v}}, S_{l}\right) ;\left(C_{\bar{v}}, C_{l}\right)$, that is, the entanglement between spins and chiralities. The fact that $\operatorname{Tr}\left[\rho_{S_{\bar{v}} S_{l}}^{2}(t)\right]<1$ indicates that the entanglement initially encoded only between the spins redistributes into spin-chirality entanglement.

Figure 3 depicts the average chirality of the lepton (a), of the antineutrino (b), and the spin-spin entanglement (c) as a function of the momentum $p$ and of the time. The spinspin entanglement oscillations depend on the chiral oscillations of both antineutrino and lepton. In the limit $m_{\bar{v}} \ll p$, the spin-spin entanglement vanishes, since the antineutrino has definite helicity and chirality. Furthermore, at intermediate dynamical regimes, the entanglement exhibits two oscillation frequencies: one related to the chiral oscillations of the antineutrino, and the other to the chiral oscillations of the lepton. For $p<m_{\bar{v}}$, the amplitude of the entanglement oscillations is suppressed, and the spin-spin entanglement is robust to the chiral oscillations. We therefore notice that the entanglement oscillations exhibit a resonance-like behavior as a function of the momentum: the amplitude of entanglement oscillations are enhanced in the dynamical regime $p \sim m_{\bar{v}}$.
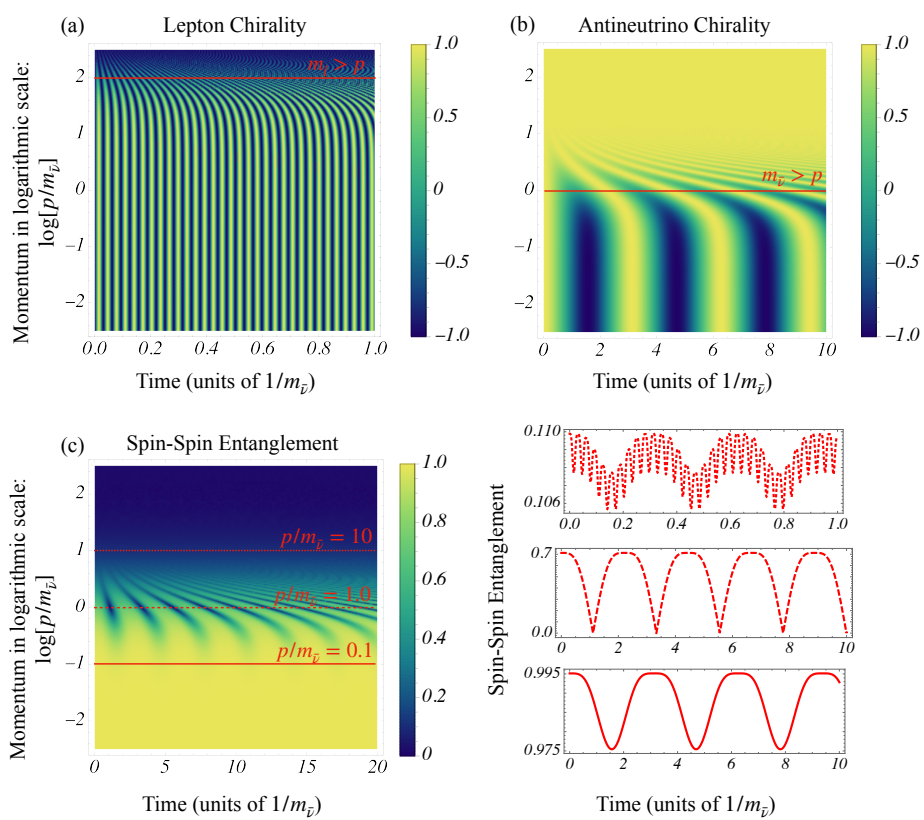

Figure 3. (a) Average lepton chirality, (b) average antineutrino chirality, and (c) spin-spin entanglement as a function of the momentum (in units of the antineutrino mass and in log scale) and of time. In (a,b) for $m_{l, \bar{v}}<p$, chiral oscillations are suppressed. Correspondingly, the spin-spin entanglement exhibits a behavior similar to the antineutrino chirality: for $m_{\bar{v}} \ll p$, entanglement is suppressed. Results for $m_{l} / m_{\bar{v}}=10^{2}$. 


\section{Chiral Oscillations on Spin Correlations}

Chiral oscillations impact the spin-spin entanglement shared between the antineutrino and the lepton. Nevertheless, the measurement of such correlations requires the full knowledge of the density matrix of the state, a task only accomplished by a tomographic reconstruction of the state.

A different approach to the problem consists of the measurement of Bell spin correlations. Since the spin-spin entanglement is modified by chiral oscillations, we expect that joint spin observables are also influenced by the chirality dynamics. In particular, we consider the following quantity:

$$
B[\rho(t)]=\left|\left\langle\hat{S}_{\bar{v}, 1} \otimes \hat{S}_{l, 1}\right\rangle+\left\langle\hat{S}_{\bar{v}, 1} \otimes \hat{S}_{l, 2}\right\rangle+\left\langle\hat{S}_{\bar{v}, 2} \otimes \hat{S}_{l, 1}\right\rangle-\left\langle\hat{S}_{\bar{v}, 2} \otimes \hat{S}_{l, 2}\right\rangle\right|,
$$

which is the Bell observable that was first proposed to investigate non-local correlations [28]. Considering

$$
\begin{aligned}
& \hat{S}_{\bar{v}, 1}=\hat{S}_{\bar{v}, x}, \quad \hat{S}_{l, 1}=-\frac{\hat{S}_{l, x}+S_{l, y}}{\sqrt{2}} \\
& \hat{S}_{\bar{v}, 2}=\hat{S}_{\bar{v}, y}, \quad \hat{S}_{l, 2}=\frac{-\hat{S}_{l, x}+S_{l, y}}{\sqrt{2}},
\end{aligned}
$$

for pure states, $B[\rho]>2$ indicates that the correlations shared between the spins are nonlocal and that the state is entangled. We notice, however, that even though for $t>0$, the spin-spin reduced density matrix is not a pure state, $B[\rho]$ still quantifies spin correlations that are affected by chiral oscillations. To quantify the total amount of non-local correlations, which does not coincide with entanglement for mixed states, one has to maximize the quantity $B$ over all possible sets of spin operators $\left\{\hat{S}_{\bar{v}, i}, \hat{S}_{l, i}\right\}$ [28]. We instead consider the simpler framework of correlations measured with the set (25). Such a type of correlations can be measured via, e.g., Stern-Gerlach apparatuses.

Figure 4 shows $B[\rho(t)]$ for the time-evolved state (15) as a function of the momentum and of time. The observable exhibits an oscillatory behavior similar to the spin-spin entanglement (c.f. Figure 3). Nevertheless, we notice that at intermediate dynamical scales (where the antineutrino is ultra-relativistic but the lepton is not), there are no clear beatings as was observed for the entanglement. Nevertheless, the imprints of chiral oscillations in $B$ are still prominent. Additionally, the Bell observable exhibits the same enhancement of oscillation amplitudes obtained for the spin-spin entanglement when the mass of the antineutrino is comparable with the momentum.
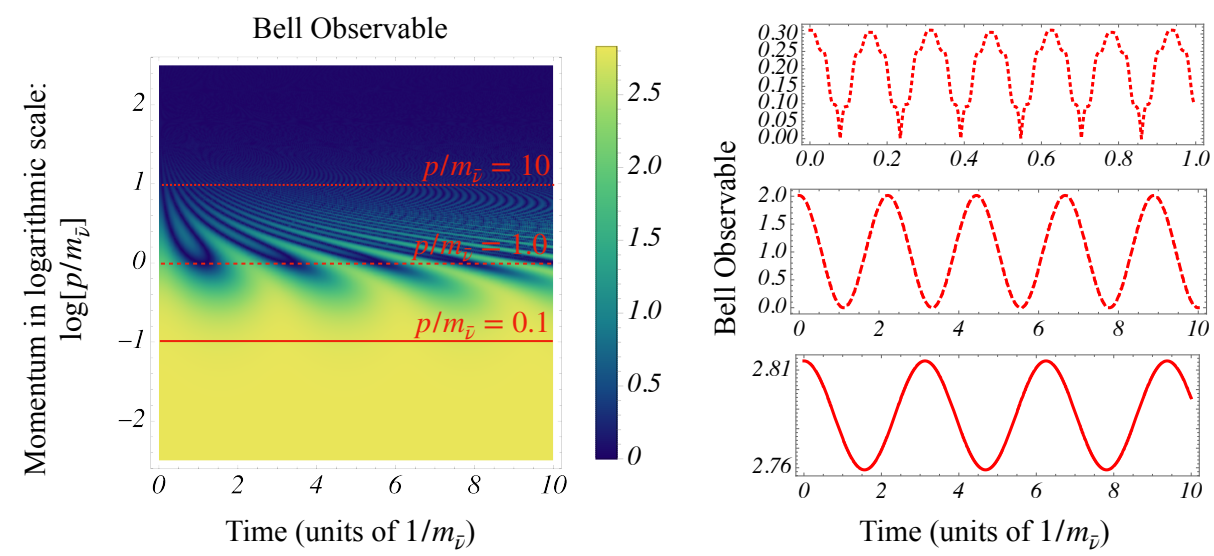

Figure 4. Bell observable (24) as a function of the momentum (in units of the antineutrino mass and in log scale) and of time for $m_{l} / m_{\bar{v}}=10^{2}$.

\section{Conclusions}

In summary, we have discussed the effects of chirality, a degree of freedom intrinsic to massive fermions described by the Dirac equation, in the spin entanglement between a 
lepton and an antineutrino. We have considered a prototypical state modeling, for example, the generation of the pair by a charged meson decay (for example, a pion). To take into account the intrinsic chiral character of weak interactions, we have further considered a chirality projection into the initial superposition.

While helicity is conserved under the Dirac equation dynamics, chirality is not. We have shown that chiral oscillations change the form of the superposition of the pair and have an imprint on the entanglement shared between the spin of the lepton and the spin of the antineutrino. The entanglement dynamics is more prominent in the case where both particles are non-relativistic, even though at intermediate dynamical regimes, the spin-spin entanglement exhibits oscillations related to both antineutrino and lepton chiral oscillations. In fact, the entanglement oscillations exhibit a resonance-like behavior with the mass of the antineutrino. Finally, we have also described how chiral oscillations are present in spin-spin correlations as measured by a Bell-like observable, where features due to chiral oscillations are present. Although we considered here a simpler scenario in which only one generation of lepton is taken into account, flavor mixing intrinsic to the dynamics of massive neutrinos can be included at the level of Dirac bispinors by following $[19,20]$. In this case, we expect a further oscillation scale related to the flavor oscillations [23] and the generation of correlations with the flavor degree of freedom [37,38]. A further step is the inclusion of wave-packets, which also influence flavor oscillations. Finally, the formalism adopted in this paper is that of single-particle relativistic quantum mechanics. A correct description of neutrino dynamics and, in particular, the inclusion of flavor oscillations requires quantum field theory [39,40].

Our results indicate a novel framework for measuring chiral oscillations, and thus the intrinsic bispinor structure of fermions, through the dynamics of either quantum entanglement, which requires a full tomography of the spin density matrix, or by spin correlations. It should be noticed that in typical pion decay experiments (for example into a muon and a muon antineutrino), the antineutrino is typically ultra-relativistic [30], and the effects reported here should be very small. Another possibility is the generation of the entangled state via scattering processes, allowing the observation of the pair in the intermediate dynamical regime, which have more prominent imprints of chirality. Finally, even though here we considered the case of an antineutrino entangled with a fermion, the framework can be readily adapted to describe chiral oscillation effects on the dynamics of two-fermion states, including fermion-antifermion pairs. This can be particularly relevant for the study of quantum correlations and entanglement in particle physics, a growing research field [41-46].

This work is a testimony of the legacy of Wigner's seminal work, which comprises several topics of physics and mathematics: from group structures and their representations to the consequences of describing quantum states with irreps of specific groups and their possible effects in physical systems.

Author Contributions: Conceptualization, V.A.S.V.B., A.E.B. and M.B.; formal analysis, V.A.S.V.B.; Writing - original draft, review and editing, V.A.S.V.B., A.E.B. and M.B. All authors have read and agreed to the published version of the manuscript.

Funding: V.A.S.V.B. acknowledges financial support from the Max Planck Gesellschaft through an Independent Max Planck Research Group.

Institutional Review Board Statement: Not Applicable.

Informed Consent Statement: Not Applicable.

Data Availability Statement: All plots presented in this paper were done using the formulas derived in the text with the corresponding parameters showed in the plot legends.

Conflicts of Interest: The authors declare no conflict of interest.

\section{References}

1. Wigner, E. On unitary representations of the inhomogeneous Lorentz Group. Ann. Math. 1939, 40, 149. [CrossRef]

2. Tung, W.K. Group Theory, 1st ed.; Cambridge University Press: New York, NY, USA, 2003.

3. Weinberg, S. Quantum Theory of Fields Vol. 1, 1st ed.; Cambridge University Press: New York, NY, USA, 1995. 
4. Peres, A.; Scudo, P.F.; Terno, D.R. Quantum Entropy and Special Relativity. Phys. Rev. Lett. 2002, 88, 230402. [CrossRef]

5. Gingrich, R.M.; Adami, C. Quantum Entanglement of Moving Bodies. Phys. Rev. Lett. 2002, 89, 270402. [CrossRef]

6. Jordan, T.F.; Shaji, A.; Sudarshan, E.C.G. Lorentz transformations that entangle spins and entangle momenta. Phys. Rev. A 2007, 75, 022101. [CrossRef]

7. Friis, N.; Bertlmann, R.A.; Huber, M.; Hiesmayr, B.C. Relativistic entanglement of two massive particles. Phys. Rev. A 2010, 81, 042114. [CrossRef]

8. Jozsa, R.; Abrams, D.S.; Dowling, J.P.; Williams, C.P. Quantum Clock Synchronization Based on Shared Prior Entanglement. Phys. Rev. Lett. 2000, 85, 2010. [CrossRef]

9. Giovannetti, V.; Lloyd, S.; Maccone, L. Quantum-enhanced positioning and clock synchronization. Nature 2001, 412, 417-419. [CrossRef] [PubMed]

10. Kent, A.; Mundro, W.J.; Spiller, P. Quantum tagging: Authenticating location via quantum information and relativistic signaling constraints. Phys. Rev. A 2011, 84, 012326. [CrossRef]

11. Mizrahi, S.S. From qubits and actions to the Pauli-Schrödinger equation. Phys. Scr. 2009, T135, 014007. [CrossRef]

12. Bernardini, A.E.; Mizrahi, S.S. Relativistic dynamics compels a thermalized fermi gas to a unique intrinsic parity eigenstate. Phys. Scr. 2014, 89, 075105. [CrossRef]

13. Bittencourt, V.A.S.V.; Bernardini, A.E.; Blasone, M. Global Dirac bispinor entanglement under Lorentz boosts. Phys. Rev. A 2018, 97, 032106. [CrossRef]

14. Bittencourt, V.A.S.V.; Bernardini, A.E. Entanglement of Dirac bi-spinor states driven by Poincaré classes of $S U(2) \otimes S U(2)$ coupling potentials. Ann. Phys. (N. Y.) 2016, 364, 182. [CrossRef]

15. Bittencourt, V.A.S.V.; Bernardini, A.E. Lattice-layer entanglement in Bernal-stacked bilayer graphene. Phys. Rev. B 2017, 95, 195145. [CrossRef]

16. Pal, P.B. Dirac, Majorana, and Weyl fermions. Am. J. Phys. 2011, 79, 485. [CrossRef]

17. De Leo, S.; Rotelli, P. Neutrino Chiral Oscillations. Int. J. Theor. Phys. 1998, 37, 2193. [CrossRef]

18. Bernardini, A.E. Chiral oscillations in terms of the zitterbewegung effect. Eur. Phys. J. C 2007, 50, 673-678. [CrossRef]

19. Bernardini, A.E.; De Leo, S. Flavor and chiral oscillations with Dirac wave packets. Phys. Rev. D 2005, 71, 076008. [CrossRef]

20. Nishi, C.C. First quantized approaches to neutrino oscillations and second quantization. Phys. Rev. D 2006, 73, 053013. [CrossRef]

21. Bernardini, A.E.; Guzzo, M.M.; Nishi, C. Quantum flavor oscillations extended to the Dirac theory. Fortschr. Phys. 2011, 59, 372. [CrossRef]

22. Cheng, T.-P.; Li, L.-F. Gauge Theory of Elementary Particle Physics, 1st ed.; Oxford Science Publication: Oxford, UK, 1995.

23. Bittencourt, V.A.S.V.; Bernardini, A.E.; Blasone, M. Chiral oscillations in the non-relativistic regime. Eur. Phys. J. C 2021, 81, 411. [CrossRef]

24. Long, A.J.; Lunardini, C.; Sabancilar, E. Detecting non-relativistic cosmic neutrinos by capture on tritium: Phenomenology and physics potential. J. Cosmol. Astropart. Phys. 2014, 1408, 038. [CrossRef]

25. Roulet, E.; Vissani, F. On the capture rates of big bang neutrinos by nuclei within the Dirac and Majorana hypotheses. J. Cosmol. Astropart. Phys. 2018, 1810, 49. [CrossRef]

26. Ge, S.-F.; Pasquini, P. Parity violation and chiral oscillation of cosmological relic neutrinos. Phys. Lett. B 2020, $811,135961$. [CrossRef]

27. Horodecki, R.; Horodecki, P.; Horodecki, M.; Horodecki, K. Quantum Entanglement. Rev. Mod. Phys. 2009, 81, 865. [CrossRef]

28. Brunner, N.; Cavalcanti, D.; Pironio, S.; Scarani, V.; Whener, S. Bell nonlocality. Rev. Mod. Phys. 2014, 86, 419. [CrossRef]

29. Thaller, B. The Dirac Equation, 1st ed.; Springer: Berlin, Germany, 1992.

30. da Motta, H. Chirality and neutrinos, a student first approach. J. Phys. Conf. Ser. 2020, 1558, 012014. [CrossRef]

31. Adam, A.S.; Benoit, N.J.; Kawamura, Y.; Matsuo, Y.; Morozumi, T.; Shimizu, Y.; Tokunaga, Y.; Toyota, N. Time evolution of lepton number carried by Majorana neutrinos. arXiv 2021, arXiv:2101.07751.

32. Pal, P.B. An Introductory Course of Particle Physics, 1st ed.; CRC Press: New York, NY, USA, 2015.

33. Peres, A. Separability criterion for density matrices. Phys. Rev. Lett. 1996, 77, 1413-1415. [CrossRef]

34. Vidal, G.; Wenerm, R.F. Computable measure of entanglement. Phys. Rev. A 2002, 65, 032314. [CrossRef]

35. Greiner, W. Relativistic Quantum Mechanics: Wave Equations, 3rd ed.; Springer: Berlin, Germany, 2000.

36. Breuer, H.-P.; Petruccionem, F. The Theory of Open Quantum Systems, 1st ed.; Oxford University Press: Oxford, UK, 2002.

37. Blasone, M.; Dell'Anno, F.; De Siena, S.; Di Mauro, M.; Illuminati, F. Multipartite entangled states in particle mixing. Phys. Rev. D 2008, 77, 096002. [CrossRef]

38. Blasone, M.; Dell'Anno, F.; De Siena, S.; Illuminati, F. Entanglement in neutrino oscillations. Europhys. Lett. $2009,85,50002$. [CrossRef]

39. Blasone, M.; Vitiello, G. Quantum field theory of fermion mixing. Ann. Phys. 1995, 244, 238-311. [CrossRef]

40. Blasone, M.; Henning, P.A.; Vitiello, G. The exact formula for neutrino oscillations. Phys. Lett. B 1999, 451, 140-145. [CrossRef]

41. Bramon, A.; Garbarino, G. Novel Bell's inequalities for entangled $K^{0} \bar{K}^{0}$ pairs. Phys. Rev. Lett. 2003, 88, 040403. [CrossRef]

42. Shi, Y. Entanglement in relativistic quantum field theory. Phys. Rev. D 2004, 70, 105001. [CrossRef]

43. Fan, J.; Li, X. Relativistic effect of entanglement in fermion-fermion scattering. Phys. Rev. D 2018, 97, 016011. [CrossRef]

44. Tanabashi, M.; Hagiwara, K.; Hikasa, K.; Nakamura, K.; Sumino, Y.; Takahashi, F.; Tanaka, J.; Agashe, K.; Aielli, G.; Amser, C.; et al. (Particle Data Group) Review of Particle Physics. Phys. Rev. D 2018, 98, 030001. [CrossRef] 
45. Afik, Y.; de Nova, J.R.M. Quantum information and entanglement with top quarks at LHC. arXiv 2020, arXiv:2003.02280.

46. Blasone, M.; De Siena, S.; Matrella, C. Wave packet approach to quantum correlations in neutrino oscillations. Eur. Phys. J. C 2021, 81,660 . 